\title{
Structural properties of the Jagla fluid
}

\author{
Mariano López de Hard* \\ Instituto de Energías Renovables, Universidad Nacional Autónoma de México (U.N.A.M.), Temixco, Morelos 62580, Mexico

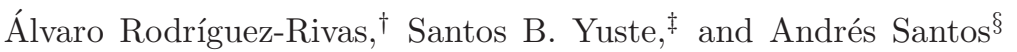 \\ Departamento de Física and Instituto de Computación Científica Avanzada (ICCAEx), \\ Universidad de Extremadura, Badajoz, E-06006, Spain
}

(Dated: August 1, 2018)

\begin{abstract}
The structural properties of the Jagla fluid are studied by Monte Carlo (MC) simulations, numerical solutions of integral equation theories, and the (semi-analytical) rational-function approximation (RFA) method. In the latter case, the results are obtained from the assumption (supported by our MC simulations) that the Jagla potential and a potential with a hard core plus an appropriate piecewise constant function lead to practically the same cavity function. The predictions obtained for the radial distribution function, $g(r)$, from this approach are compared against $\mathrm{MC}$ simulations and integral equations for the Jagla model, and also for the limiting cases of the triangle-well potential and the ramp potential, with a general good agreement. The analytical form of the RFA in Laplace space allows us to describe the asymptotic behavior of $g(r)$ in a clean way and compare it with MC simulations for representative states with oscillatory or monotonic decay. The RFA predictions for the Fisher-Widom and Widom lines of the Jagla fluid are obtained.
\end{abstract}

\section{INTRODUCTION}

Substances such as water, phosphorous, carbon, or silicon, whose intermolecular interactions depend on the orientation between their bonds, can all exhibit anomalous static and/or dynamic behavior. In order to understand and deal with such behavior, it is convenient to rely on simple models which may shed light on the mechanisms leading to it. Ever since the appearance of the pioneering work by Hemmer and Stell [1, 2] on fluid systems whose molecules interact through spherically symmetric coresoftened pair potentials, these and closely related models have been considered in the literature for the previous purpose. Among the problems that have been addressed, one finds such models in attempts to describe reentrant melting [3 8 ], thermodynamic anomalies [3, 9-43], anomalous transport [34, 41, 43 49], liquid-liquid phase transitions and phase diagrams 21, 33, 36, 42, 50 74, and glassy behavior $11,17,20,26,28,49,53,67,70,75-$ 77].

Among the family of core-softened pair potentials, one that is able to predict multiple fluid transitions and some of the water-type thermodynamic and dynamic anomalies is the Jagla ramp potential (hard core plus a linear repulsive ramp and a linear attractive ramp) [11] given

\footnotetext{
* malopez@unam.mx http://xml.cie.unam.mx/xml/tc/ft/mlh/

$\dagger$ arodriguezrivas@unex.es https://arodriguez-rivas.weebly.com/

$¥$ santos@unex.es http://www.unex.es/eweb/fisteor/santos/

$\S$ andres@unex.es http://www.unex.es/eweb/fisteor/andres/
}

by

$$
\phi(r)= \begin{cases}\infty, & r<\sigma, \\ \frac{\varepsilon_{1}\left(\lambda_{1}-r\right)-\varepsilon_{2}(r-\sigma)}{\lambda_{1}-\sigma}, & \sigma \leq r \leq \lambda_{1}, \\ -\frac{\varepsilon_{2}\left(\lambda_{2}-r\right)}{\lambda_{2}-\lambda_{1}}, & \lambda_{1} \leq r \leq \lambda_{2}, \\ 0, & r \geq \lambda_{2} .\end{cases}
$$

The explicit form of this potential is determined by five parameters, namely three lengths (the hard-core diameter $\sigma$ and the ranges $\lambda_{1}$ and $\lambda_{2}$ ) and two energies (the height $\varepsilon_{1}$ of the repulsive ramp and the depth $\varepsilon_{2}$ of the attractive well, both taken to be positive). It includes the two interesting limiting cases of the triangle-well potential $\left(\lambda_{1}=\sigma, \lambda_{2}=\lambda, \varepsilon_{2}=\varepsilon\right)$ and the ramp potential $\left(\varepsilon_{2}=0, \varepsilon_{1}=\varepsilon, \lambda_{1}=\lambda_{2}=\lambda\right)$. Figures 1(a)-1(c) sketch the Jagla, triangle-well, and ramp potentials, respectively.

For such fluids, we take $\sigma=1$ as the length unit and $\varepsilon_{\text {ref }}=\varepsilon_{2}=1$ (Jagla potential), $\varepsilon_{\text {ref }}=\varepsilon=1$ (trianglewell potential), or $\varepsilon_{\text {ref }}=\varepsilon=1$ (ramp potential) as the energy unit. Reduced units ( $\rho^{*}=\rho \sigma^{3}$ for the density and $T^{*}=k_{B} T / \varepsilon_{\text {ref }}$ for the temperature) are used, so that the additional relevant parameters are $\varepsilon_{1} / \varepsilon_{2}, \lambda_{1}$, and $\lambda_{2}$ for the Jagla potential, and $\lambda$ for the triangle-well and ramp potentials.

While there has been a lot of work in the literature concerning the thermodynamic properties of the Jagla fluid, as far as we know no work other than the paper by Gibson and Wilding [60] has been devoted to the structural properties of a fluid whose molecules interact through such a potential. Therefore, the major aim of this paper is to present a semi-analytical approach based on the rationalfunction approximation (RFA) 78 80] to obtain the radial distribution function $g(r)$ of the Jagla fluid, including its asymptotic behavior for large $r$. The application of the RFA to the Jagla fluid is made by assuming that a 


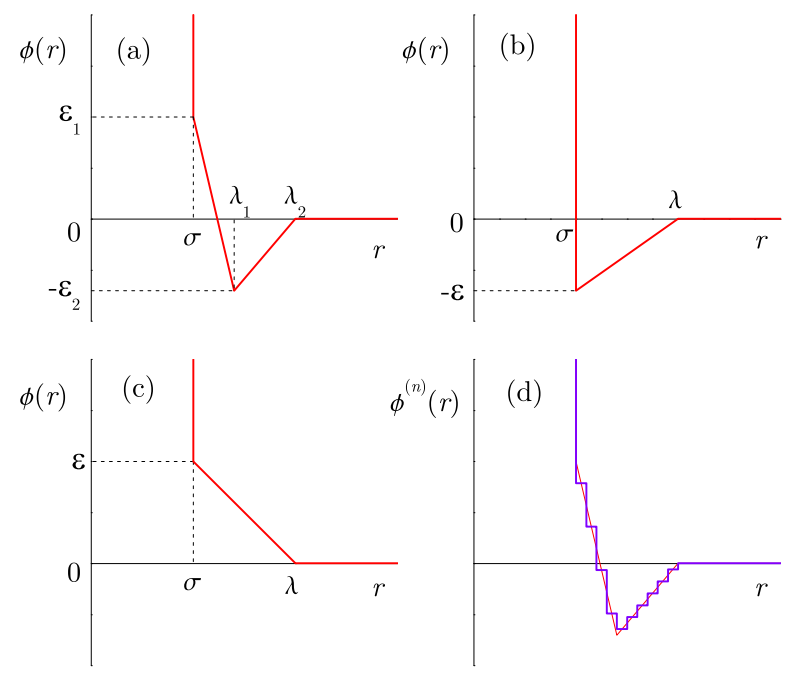

FIG. 1. Sketch of (a) the Jagla potential, (b) the trianglewell potential, and (c) the ramp potential. (d) A sketch of the discretized version of the Jagla potential with $n=10$.

representation of the potential consisting in a hard core plus an appropriate piecewise constant function leads to essentially the same cavity function as the original Jagla potential. The outcome of the RFA approach will be assessed by testing its validity against integral equation results [both the Percus-Yevick (PY) and hypernettedchain (HNC) approximations will be considered] and our own Monte Carlo (MC) simulation data.

The paper is organized as follows. Section $\Pi$ discusses how the radial distribution function of the Jagla fluid can be approximately obtained from that of a discretized $n$-step potential. Next, in order to make the paper selfcontained, in Sec. III we summarize the RFA method leading to the structural properties of the $n$-step fluid. This is followed in Sec. IV] by the comparison between the results obtained with the present approach for various cases of Jagla, triangle-well, and ramp fluids, and those of the PY and HNC approximations when tested against our MC simulation data. Section $\mathrm{V}$ deals with the Fisher-Widom and Widom lines, as well as with the static structure factor, of the Jagla fluid. The paper is closed in Sec. VI with some discussion and concluding remarks.

\section{MAPPING TO PIECEWISE CONSTANT POTENTIALS}

Suppose we want to replace the actual potential (10) by a discretized version $\phi^{(n)}(r)$ consisting of a sequence of $n$ steps of "heights" $\epsilon_{j}$ and widths $\left(\ell_{j}-\ell_{j-1}\right)$ (with the conventions $\ell_{0}=\sigma$ and $\ell_{n}=\lambda_{2}$ ), namely [70, 81]

$$
\phi^{(n)}(r)= \begin{cases}\infty, & r<\sigma, \\ \epsilon_{1}, & \sigma<r<\ell_{1}, \\ \epsilon_{2}, & \ell_{1}<r<\ell_{2}, \\ \vdots & \vdots \\ \epsilon_{n}, & \ell_{n-1}<r<\ell_{n}, \\ 0, & r>\ell_{n} .\end{cases}
$$

The simplest choice for $\phi^{(n)}(r)$ to mimic the original potential $\phi(r)$ is to take $\epsilon_{j}=\phi\left(\left(\ell_{j-1}+\ell_{j}\right) / 2\right)$ and a constant step width $\Delta r=\left(\lambda_{2}-\sigma\right) / n$, so that $\ell_{j}=\sigma+j \Delta r$. This is illustrated in Fig. 1(d) for the case $n=10$.

Once the representation $\phi(r) \rightarrow \phi^{(n)}(r)$ has been done, the next step in the mapping consists in approximating the cavity function,

$$
y(r) \equiv g(r) e^{\beta \phi(r)}, \quad \beta \equiv \frac{1}{k_{B} T},
$$

of the Jagla fluid with the one of the $n$-step fluid, $y^{(n)}(r) \equiv g^{(n)}(r) e^{\beta \phi^{(n)}(r)}$, namely

$$
y(r) \approx y^{(n)}(r)
$$

or, equivalently,

$$
g(r) \approx g^{(n)}(r) e^{\beta\left[\phi^{(n)}(r)-\phi(r)\right]} .
$$

Clearly, the practical usefulness of the method rests on considering a relatively small number of steps in the discretization.

While the MC results that are presented in Secs. IV and $\mathrm{V}$ have been obtained for the true Jagla potential (1), we have performed additional MC simulations for the discretized potential (2), with different choices of $n$, in order to test the reliability of the ansatz (4).

For illustration purposes, in Figs. 2(a) and 2(b) we show, respectively, the cavity functions $y(r)$ and $y^{(n)}(r)$, and the deviations $y^{(n)}(r)-y(r)$ (with $n=5, n=10$, $n=15$, and $n=20$ ) for a representative Jagla fluid. Here, the potential parameters correspond to $\lambda_{1}=1.3$, $\lambda_{2}=1.6$, and $\varepsilon_{2}=\varepsilon_{1}$, while the reduced density and temperature are $\rho^{*}=0.6$ and $T^{*}=1$, respectively. One can observe in Fig. 2 that the functions $y^{(n)}(r)$ are practically indistinguishable from the true Jagla cavity function $y(r)$. It is also clear that the cavity function $y^{(n)}(r)$ with $n=5$ underestimates the contact value and the values up to the first minimum, while such limitations are widely corrected with $n=10, n=15$ and, especially, $n=20$. To the best of our knowledge, the strong insensitivity of the cavity function to the "details" of the potential (such as the number of steps), and hence the practical validity of Eqs. (4) and (5), has not been noted before.

Nevertheless, a word of caution and some technical issues are pertinent here, since not everything is as clearcut as the previous analysis could suggest. Paradoxically, 


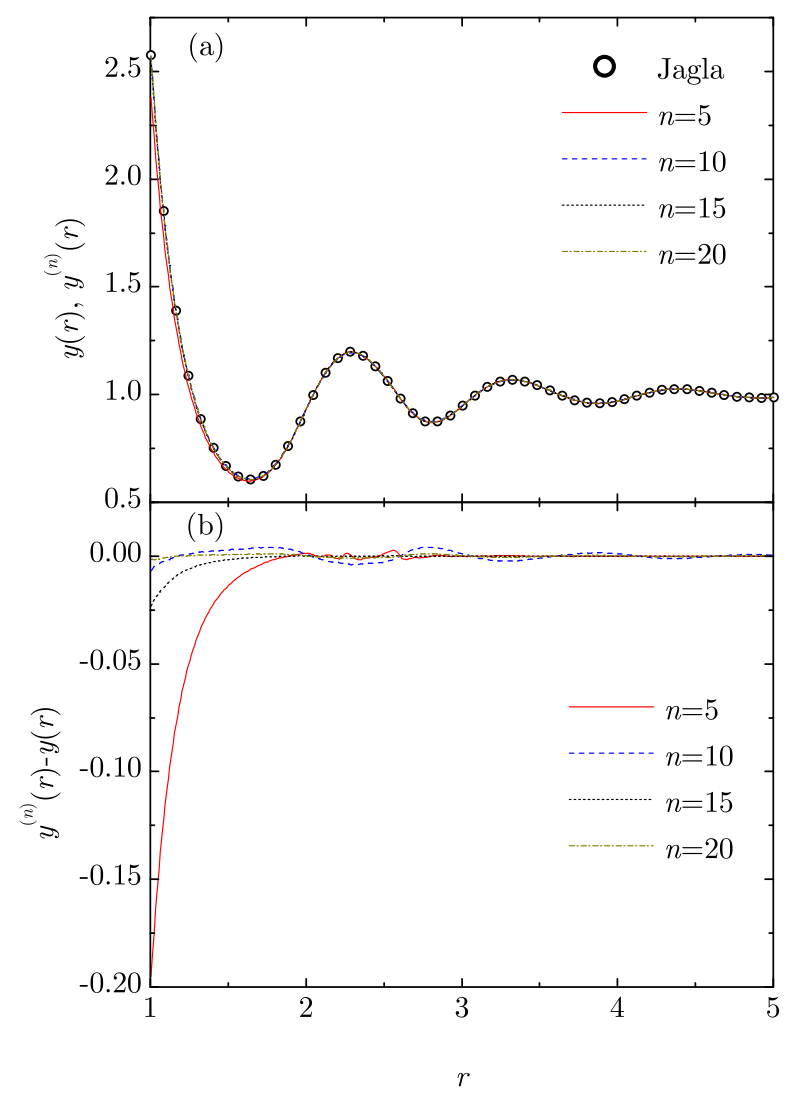

FIG. 2. (a) MC results for the cavity function at $\rho^{*}=0.6$ and $T^{*}=1$. The circles correspond to the true Jagla potential with $\lambda_{1}=1.3, \lambda_{2}=1.6$, and $\varepsilon_{1} / \varepsilon_{2}=1$, while the lines correspond to the discretized version of the same potential with $n=5(-), n=10(--), n=15(\cdots)$, and $n=20$ (-.-.-). (b) Difference between the MC cavity function of the discretized potential and the MC cavity function of the genuine Jagla potential.

the differences $y^{(n)}(r)-y(r)$ with $n=10$ are typically smaller than with $n=15$. This shows that the convergence $y^{(n)}(r) \rightarrow y(r)$ is not monotonic with increasing $n$. In particular, we have observed that in the case $\left(\lambda_{1}, \lambda_{2}\right)=(1.3,1.6)$ the optimal values are $n=$ even, but other choices are more appropriate for other values of $\left(\lambda_{1}, \lambda_{2}\right)$.

A convenient predictor of the optimal values of $n$ relies on the use of the second virial coefficient [80, 82]

$$
B_{2}=-2 \pi \int_{0}^{\infty} d r r^{2}\left[e^{-\beta \phi(r)}-1\right] .
$$

Our MC simulations suggest that a reliable criterion to predict the qualitative dependence on $n$ of the difference between $y^{(n)}(r)$ and $y(r)$ is based on the analysis of the relative difference $\delta B_{2}^{(n)} \equiv\left|B_{2}^{(n)} / B_{2}-1\right|$ between the second virial coefficient, $B_{2}^{(n)}$, of the discretized potential $\phi^{(n)}(r)$ and the second virial coefficient, $B_{2}$, of the continuous potential $\phi(r)$. Figure 3 shows that $\delta B_{2}^{(n)}$

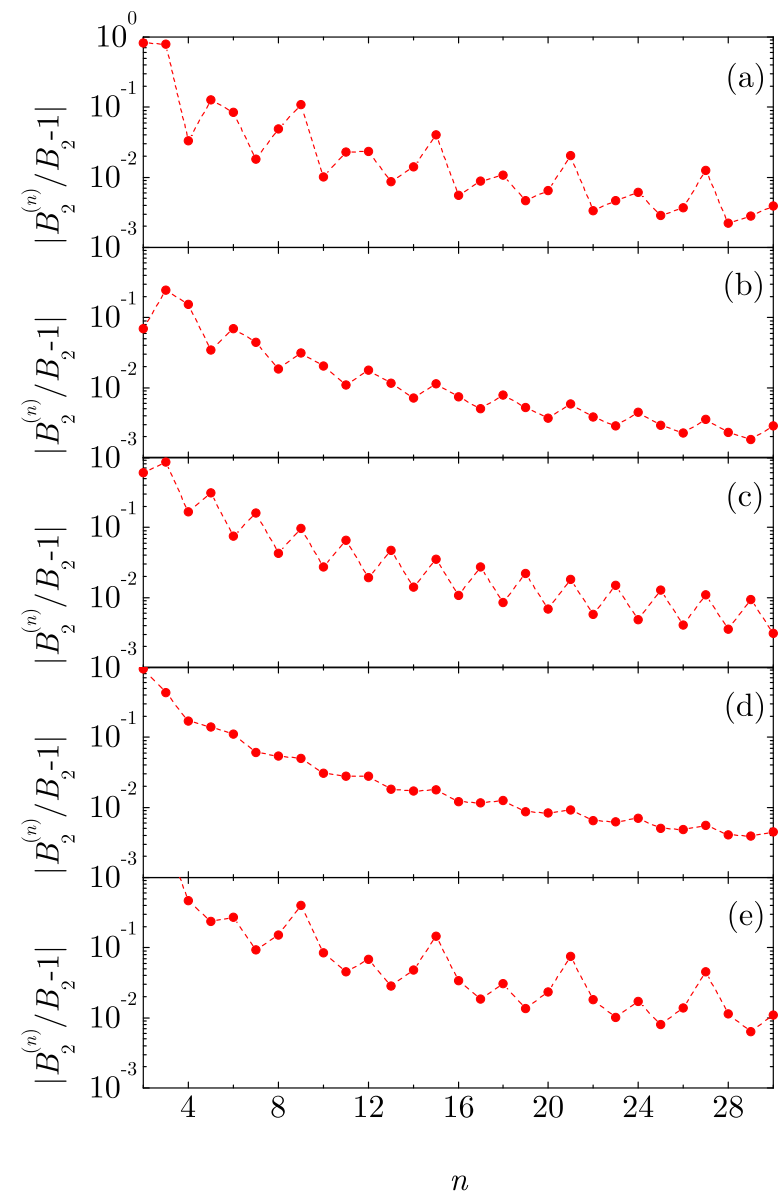

FIG. 3. Relative difference $\left|B_{2}^{(n)} / B_{2}-1\right|$ versus the number of steps $n$ for the Jagla potential with $\varepsilon_{1} / \varepsilon_{2}=1, T^{*}=1$, $\lambda_{2}=1.6$, and (a) $\lambda_{1}=1.1$, (b) $\lambda_{1}=1.2$, (c) $\lambda_{1}=1.3$, (d) $\lambda_{1}=1.4$, and (e) $\lambda_{1}=1.5$. The lines are a guide to the eye.

presents local minima at certain (optimal) values of $n$, which follow regular patterns depending on the values of $\left(\lambda_{1}, \lambda_{2}\right)$. For instance, in the case of Fig. 3(e), one can observe that the choice $n=13$ is better than the higher values $n=14,15$, and 16 . We have checked that the qualitative dependence of $\delta B_{2}^{(n)}$ on $n$ is not affected by the values of $T^{*}$ and $\varepsilon_{1} / \varepsilon_{2}$. Finally, although not shown, it should be stressed that the convergence with increasing $n$ is monotonic in the cases of the ramp and triangle-well potentials. In any case, the choice $n=10$ seems to be a reasonable one in most instances.

\section{RFA APPROACH FOR PIECEWISE CONSTANT POTENTIALS WITH A HARD CORE}

In this section we provide the essential steps leading to the computation of the radial distribution function $g^{(n)}(r)$ of the fluid with the $n$-step potential (2). For further details, we refer the reader to Refs. 78 80. We 
begin by expressing the Laplace transform of $r g^{(n)}(r)$ as

$$
G^{(n)}(s)=s \frac{F^{(n)}(s) e^{-s}}{1+12 \eta F^{(n)}(s) e^{-s}},
$$

where $\eta \equiv \frac{\pi}{6} \rho^{*}$ is the packing fraction. Further, $F^{(n)}(s)$ is decomposed as

$$
F^{(n)}(s)=\sum_{j=0}^{n} R_{j}^{(n)}(s) e^{-\left(\ell_{j}-1\right) s}
$$

to reflect the discontinuities of $g^{(n)}(r)$ at $r=\ell_{j}$.

We next assume the following rational-function approximation for $R_{j}^{(n)}(s)$ :

$$
\begin{gathered}
R_{j}^{(n)}(s)=-\frac{1}{12 \eta} \frac{a_{j}^{(n)}+b_{j}^{(n)} s}{1+\mathcal{S}_{1}^{(n)} s+\mathcal{S}_{2}^{(n)} s^{2}+\mathcal{S}_{3}^{(n)} s^{3}}, \\
j=0, \ldots, n .
\end{gathered}
$$

The $2 n+5$ constants $\left\{a_{j}^{(n)}, b_{j}^{(n)} ; j=0, \ldots, n\right\}$ and $\left\{\mathcal{S}_{k}^{(n)} ; k=1,2,3\right\}$ must satisfy certain consistency conditions. First, the exact physical requirement $G^{(n)}(s)=$ $s^{-2}+O\left(s^{0}\right)$ implies

$$
\begin{gathered}
a_{0}^{(n)}=1-\sum_{j=1}^{n} a_{j}^{(n)}, \\
b_{0}^{(n)}=c_{1}^{(n)}+\frac{\eta / 2}{1+2 \eta}\left[6 c_{2}^{(n)}+4 c_{3}^{(n)}+c_{4}^{(n)}\right]+\frac{1+\eta / 2}{1+2 \eta}, \\
\mathcal{S}_{1}^{(n)}=b_{0}^{(n)}-c_{1}^{(n)}-1 \\
\mathcal{S}_{2}^{(n)}=\frac{1}{2}-b_{0}^{(n)}+c_{1}^{(n)}+\frac{1}{2} c_{2}^{(n)} \\
\mathcal{S}_{3}^{(n)}=\frac{1}{2} b_{0}^{(n)}-\frac{1}{2} c_{1}^{(n)}-\frac{1}{2} c_{2}^{(n)}-\frac{1}{6} c_{3}^{(n)}-\frac{1+2 \eta}{12 \eta}
\end{gathered}
$$

where

$$
c_{k}^{(n)} \equiv \sum_{j=1}^{n}\left[a_{j}^{(n)}\left(\ell_{j}-1\right)^{k}-k b_{j}^{(n)}\left(\ell_{j}-1\right)^{k-1}\right] .
$$

Thus, the five coefficients $a_{0}^{(n)}, b_{0}^{(n)}, \mathcal{S}_{1}^{(n)}, \mathcal{S}_{2}^{(n)}$, and $\mathcal{S}_{3}^{(n)}$ are linear combinations of the $2 n$ parameters $\left\{a_{j}^{(n)}, b_{j}^{(n)} ; j=1, \ldots, n\right\}$. Next, one must account for the fact that the cavity function $y^{(n)}(r)$ is continuous at $r=\ell_{j}$. Specifically,

$$
\begin{aligned}
\frac{b_{j}^{(n)}}{\mathcal{S}_{3}^{(n)}}= & \sum_{\alpha=1}^{3} \frac{\left[e^{\beta\left(\epsilon_{j}-\epsilon_{j+1}\right)}-1\right] s_{\alpha}^{(n)} e^{\ell_{j} s_{\alpha}^{(n)}}}{\mathcal{S}_{1}^{(n)}+2 \mathcal{S}_{2}^{(n)} s_{\alpha}^{(n)}+3 \mathcal{S}_{3}^{(n)} s_{\alpha}^{(n) 2}} \\
& \times \sum_{i=0}^{j-1}\left[a_{i}^{(n)}+b_{i}^{(n)} s_{\alpha}^{(n)}\right] e^{-\ell_{i} s_{\alpha}^{(n)}}, \quad j=1, \ldots, n,
\end{aligned}
$$

with the convention $\epsilon_{n+1}=0$ and where $s_{\alpha}^{(n)}(\alpha=1,2,3)$ are the three roots of the cubic equation $1+\mathcal{S}_{1}^{(n)} s+$ $\mathcal{S}_{2}^{(n)} s^{2}+\mathcal{S}_{3}^{(n)} s^{3}=0$. Finally, for simplicity, the parameters $\left\{a_{j}^{(n)}\right\}$ are set to their low-density values, namely

$$
a_{j}^{(n)}=e^{-\beta \epsilon_{j+1}}-e^{-\beta \epsilon_{j}}, \quad j=1, \ldots, n .
$$

Insertion of Eqs. (10) and (13) into Eq. (12) yields a set of $n$ coupled transcendental equations for $\left\{b_{j}^{(n)} ; j=\right.$ $1, \ldots, n\}$ that has to be solved numerically. This task can be undertaken with the help of a computer algebra system and turns out to be rather manageable, even for $n=20$. Once the solution is found, Eqs. (7)-(9) provide the explicit $s$ dependence of the Laplace transform $G^{(n)}(s)$. Laplace inversion of Eq. (7) yields a useful representation of $g^{(n)}(r)$ as

$$
g^{(n)}(r)=r^{-1} \sum_{m=1}^{\infty}(-12 \eta)^{m-1} f_{m}^{(n)}(r-m) \Theta(r-m),
$$

where $f_{m}^{(n)}(r)$ is the inverse Laplace transform of $s\left[F^{(n)}(s)\right]^{m}$ and $\Theta(r)$ is the Heaviside step function. In particular, the contact value is

$$
g^{(n)}\left(1^{+}\right)=\lim _{s \rightarrow \infty} s^{2} F^{(n)}(s)=\frac{b_{0}^{(n)}}{\mathcal{S}_{3}^{(n)}} .
$$

We have checked that the dependence of $y^{(n)}(1)$ on $n$ follows essentially the same pattern as the second virial coefficient $B_{2}^{(n)}$.

Once the RFA provides the radial distribution function $g^{(n)}(r)$ for the $n$-step potential (2), the ansatz (5) allows one to obtain the RFA prediction for the continuous potential.

\section{RADIAL DISTRIBUTION FUNCTION. MC SIMULATIONS AND THEORETICAL RESULTS}

In this section we present the results of the computations performed with the RFA approach and those of NVT MC simulations of a fluid of particles that interact with the Jagla, triangle-well, and ramp potentials (see Fig. (1). Numerical solutions of the PY and HNC integral equations are also presented. It must be remarked that, as said before, the MC simulations were performed on the true Jagla potential (11). The same happens with the numerical solutions of the PY and HNC integral equations. On the other hand, the results corresponding to the RFA were obtained by the method described in Sec. III and application of Eq. (5).

In the $\mathrm{MC}$ simulations, we considered a system with $N=1372$ particles. The system was simulated during $10^{6}$ MC steps (each one consisting of $N$ displacement attempts) for equilibration plus $2 \times 10^{6}$ additional MC steps to collect data every $50 \mathrm{MC}$ steps, taking averages 


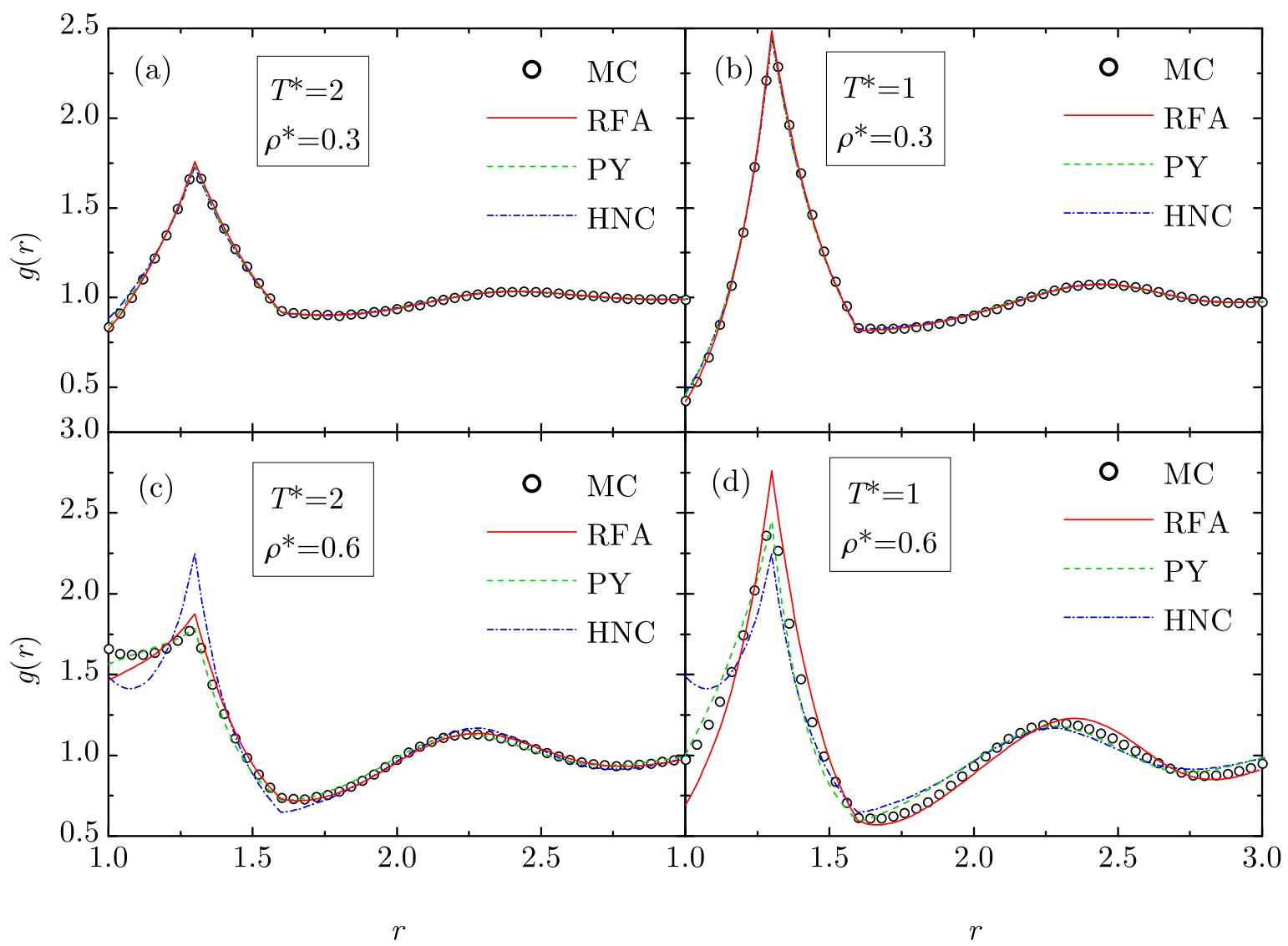

FIG. 4. Radial distribution function of a fluid with a Jagla potential $\left(\lambda_{1}=1.3, \lambda_{2}=1.6, \varepsilon_{1} / \varepsilon_{2}=1\right)$ at $(\mathrm{a})\left(T^{*}, \rho^{*}\right)=(2,0.3)$, (b) $\left(T^{*}, \rho^{*}\right)=(1,0.3)$, (c) $\left(T^{*}, \rho^{*}\right)=(2,0.6)$, and (d) $\left(T^{*}, \rho^{*}\right)=(1,0.6)$. The symbols, solid lines, dashed lines, and dashdotted lines correspond to MC simulations, the RFA with $n=10$, the PY integral equation, and the HNC integral equation, respectively.

every 1000 recorded data points. Moreover, 50 independent simulations were performed for each given case. The radial distribution function $g(r)$ was averaged over the 50 simulations and evaluated with a bin size $\delta r=0.01$.

For the sake of illustration, in what follows we have taken $\lambda=1.6$ for the triangle-well and ramp potentials, and $\lambda_{1}=1.3, \lambda_{2}=1.6$, and $\varepsilon_{1} / \varepsilon_{2}=1$ for the Jagla potential. We have chosen four representative states $\left(\rho^{*}=0.3\right.$ and $0.6, T^{*}=1$ and 2$)$. The results are displayed in Figs. 4, 6 .

It is worth pointing out that, for the cases presented, the RFA approach leads to very good agreement with the MC simulation results. In fact, such an agreement is as good as, and in some instances better than, the solution of either the PY or the HNC integral equations. This is especially noteworthy since the RFA only requires the numerical solution of a few (actually $n$ ) nonlinear equations, whereas the PY and HNC integral equations are numerically solved by introducing a cutoff distance, replacing the integrals by sums involving a large number of unknown local values, and iterating until certain convergence criteria are satisfied.

As expected [78, 79], the performance of the RFA tends to worsen as density increases and/or temperature decreases, especially near contact. On the other hand, even in those cases, the oscillations of $g(r)$ for larger distances are well accounted for, a feature that is addressed with more detail in Sec. $\nabla$.

Also remarkable is the fact that, with a relatively small number of steps (in all of these cases we took $n=10$ ), one gets a rather reasonable description of the structure of the fluid. This shows that, as confirmed by Fig. 2 , the replacement of the original potential $\phi(r)$ by a discretized version $\phi^{(n)}(r)$, and the subsequent application of Eq. (5), indeed represent a practical route to obtain the structural properties of the fluid.

\section{FISHER-WIDOM AND WIDOM LINES FOR THE JAGLA FLUID}

According to general arguments 83 86, the total correlation function $h(r) \equiv g(r)-1$ can be written as

$$
r h(r)=\sum_{i} \mathcal{A}_{i} e^{s_{i} r}=\mathcal{A}_{1} e^{s_{1} r}+\mathcal{A}_{2} e^{s_{2} r}+\mathcal{A}_{3} e^{s_{3} r}+\cdots,
$$




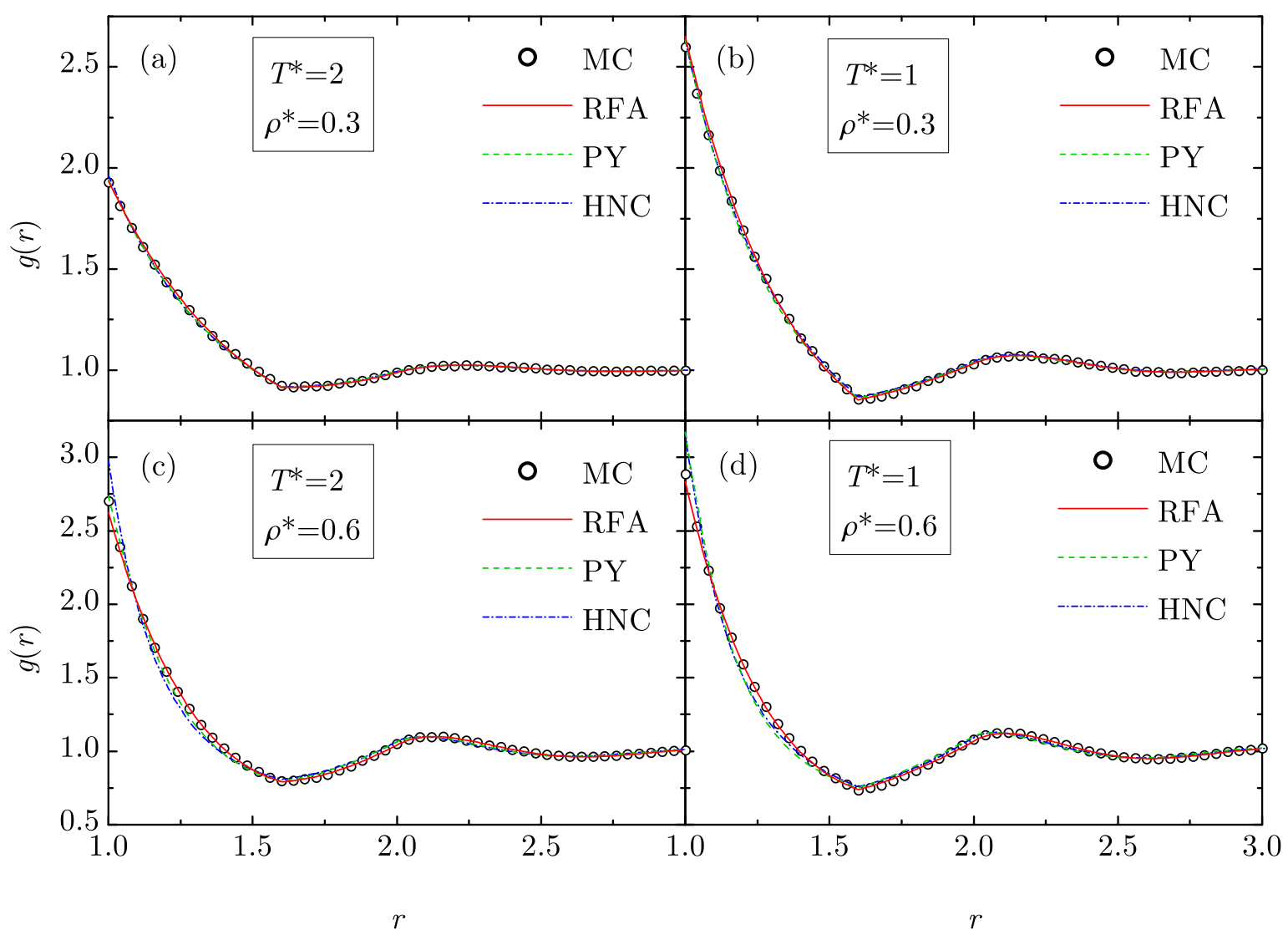

FIG. 5. Radial distribution function of a fluid with a triangle-well potential $(\lambda=1.6)$ at $(\mathrm{a})\left(T^{*}, \rho^{*}\right)=(2,0.3),(\mathrm{b})\left(T^{*}, \rho^{*}\right)=$ $(1,0.3)$, (c) $\left(T^{*}, \rho^{*}\right)=(2,0.6)$, and $(\mathrm{d})\left(T^{*}, \rho^{*}\right)=(1,0.6)$. The symbols, solid lines, dashed lines, and dash-dotted lines correspond to MC simulations, the RFA with $n=10$, the PY integral equation, and the HNC integral equation, respectively.

where the sum runs over the discrete set of nonzero poles $s_{i}$ of the Laplace transform $G(s)$ of $r g(r)$, the ordering $0>\operatorname{Re}\left(s_{1}\right) \geq \operatorname{Re}\left(s_{2}\right) \geq \operatorname{Re}\left(s_{3}\right) \geq \cdots$ is adopted, and the amplitudes $\mathcal{A}_{i}=\operatorname{Res}[G(s)]_{s_{i}}$ are the associated (in general complex) residues. The asymptotic decay of $h(r)$ is determined by the nature of the pole(s) with the largest real part.

In general, in the case of potentials with an attractive part, the three dominating terms in Eq. (16) are those associated with a pair of complex conjugate poles $s_{1,2}=$ $-\gamma \pm \imath \omega$ (or $s_{2,3}=-\gamma \pm \imath \omega$ ), where $\imath$ denotes the imaginary unit, and a real pole $s_{3}=-\kappa$ (or $s_{1}=-\kappa$ ). Thus, the dominant behavior of $h(r)$ at large $r$ is

$$
h(r) \approx \frac{1}{r} \begin{cases}2\left|\mathcal{A}_{\gamma}\right| e^{-\gamma r} \cos (\omega r+\psi), & \gamma<\kappa \\ \mathcal{A}_{\kappa} e^{-\kappa r}, & \gamma>\kappa\end{cases}
$$

where $\psi$ is the argument of the residue $\mathcal{A}_{\gamma}$, i.e., $\mathcal{A}_{\gamma}=$ $\left|\mathcal{A}_{\gamma}\right| e^{ \pm \imath \psi}$. Thus, the asymptotic behavior of $h(r)$ results from the competition between the real parts closest to the origin of the poles of $G(s)$. If $\gamma<\kappa$, a pair of conjugate complex poles dominate and the decay of the total correlation function is oscillatory. On the other hand, if $\kappa<\gamma$, a real pole is the dominant one and then the asymptotic decay is monotonic. In the latter case, $\xi=\kappa^{-1}$ repre- sents the correlation length.

The oscillatory decay reflects the effects of the repulsive part of the interaction potential on spatial pair correlations, while the effects of the attractive part are reflected by the monotonic decay. Thus, at a given temperature, the first type of decay takes place at sufficiently high values of density, whereas the monotonic decay occurs at sufficiently low values of density. Following Fisher and Widom [84], the locus of transition points from one type to the other one $(\gamma=\kappa)$ defines a line, the so-called Fisher-Widom (FW) line, in the temperatureversus-density plane. This line has been the subject of many investigations for different fluid models 83,86 , 89].

Since the RFA works, by construction, in Laplace space [see Eq. (7)] and provides an explicit dependence of $G(s)$ on $s$, it is ideally suited to determine the poles with a real part closest to the origin. As a representative example, we consider the Jagla fluid with $\lambda_{1}=1.3, \lambda_{2}=1.6$, and $\varepsilon_{1} / \varepsilon_{2}=1$. Moreover, the RFA with $n=10$ is generally employed.

Figure 7 shows the density dependence of both $\gamma$ and $\kappa$ at several temperatures. While, at a given temperature $T^{*}, \gamma$ monotonically decreases with increasing density, $\kappa$ presents a nonmonotonic behavior. As a consequence, both curves cross at a certain point of density $\rho_{\mathrm{FW}}^{*}\left(T^{*}\right)$, 


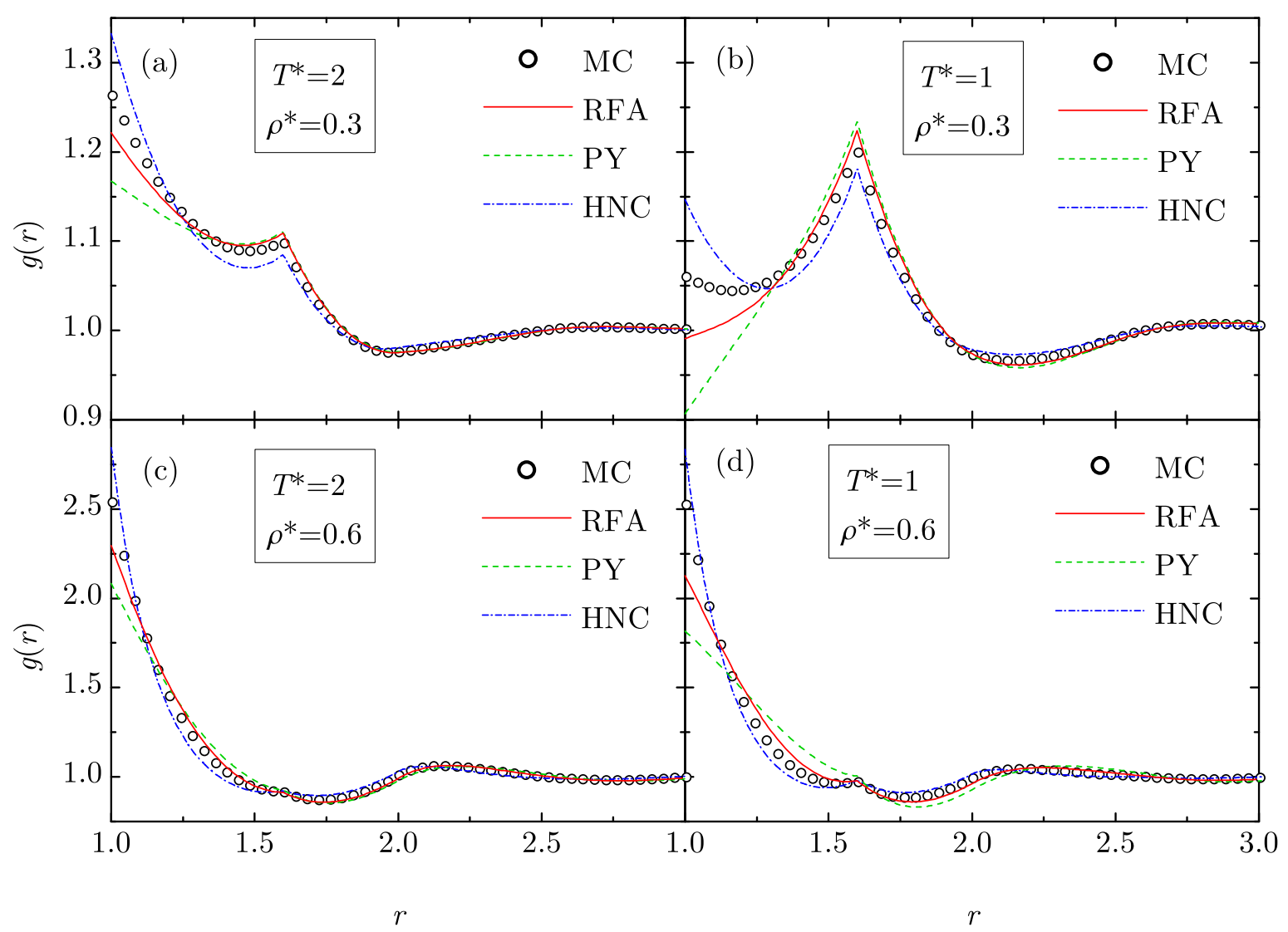

FIG. 6. Radial distribution function of a fluid with a ramp potential $(\lambda=1.6)$ at $(\mathrm{a})\left(T^{*}, \rho^{*}\right)=(2,0.3),(\mathrm{b})\left(T^{*}, \rho^{*}\right)=(1,0.3)$, (c) $\left(T^{*}, \rho^{*}\right)=(2,0.6)$, and (d) $\left(T^{*}, \rho^{*}\right)=(1,0.6)$. The symbols, solid lines, dashed lines, and dash-dotted lines correspond to MC simulations, the RFA with $n=10$, the PY integral equation, and the HNC integral equation, respectively.

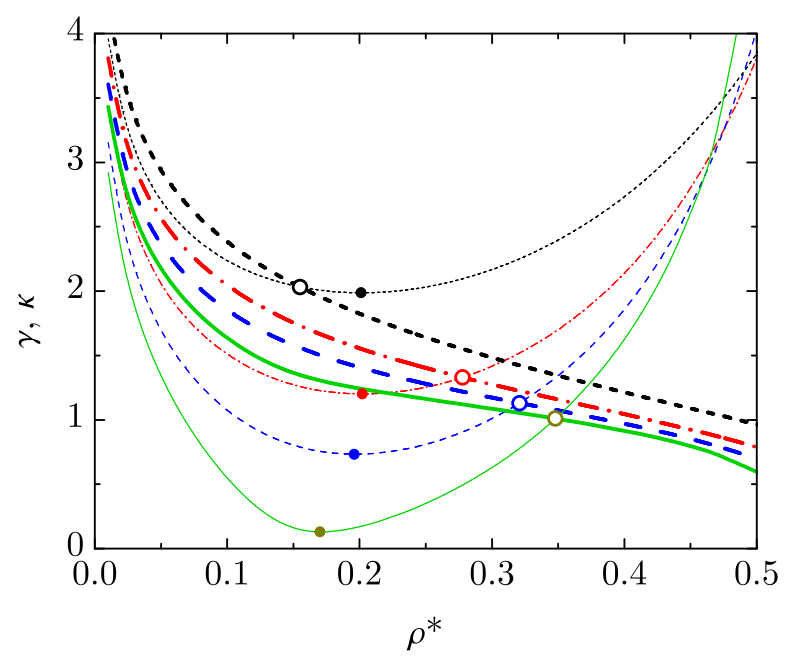

FIG. 7. Density dependence of the damping coefficients $\gamma$ (thick lines) and $\kappa$ (thin lines) in a fluid with a Jagla potential $\left(\lambda_{1}=1.3, \lambda_{2}=1.6, \varepsilon_{1} / \varepsilon_{2}=1\right)$, as predicted by the RFA $(n=10)$. The temperatures are $T^{*}=1.2(--), T^{*}=0.8$ $(-\cdot-\cdot), T^{*}=2 / 3(---)$, and $T^{*}=0.58(-)$. At each temperature, the open circle marks the intersection point $\gamma=$ $\kappa$ (FW transition point). The minima of the curves for $\kappa$ (solid circles) define the Widom line. such that $\gamma \leq \kappa$ (oscillatory decay) if $\rho^{*} \geq \rho_{\mathrm{FW}}^{*}\left(T^{*}\right)$ and $\kappa<\gamma$ (monotonic decay) if $\rho^{*}<\rho_{\mathrm{FW}}^{*}\left(T^{*}\right)$.

The locus of points $\rho_{\mathrm{FW}}^{*}\left(T^{*}\right)$ defines the $\mathrm{FW}$ line, which is plotted in Fig. 8. The FW line predicted by the RFA with $n=5$, as well as a few points obtained with $n=20$, are also shown in Fig. 8. Although qualitatively analogous, the line with $n=5$ differs from that with $n=10$. However, moving from $n=10$ to $n=20$ has practically no effect on the FW line. Since $y(r)=g(r)$ for $r>\lambda_{2}$, the excellent agreement between the FW lines with $n=10$ and $n=20$ is a fine-grained test of the ansatz (4).

The nonmonotonic behavior of $\kappa$ versus $\rho^{*}$ at fixed $T^{*}$ observed in Fig. 7 implies the occurrence of a minimum value at a certain density $\rho_{\mathrm{W}}^{*}\left(T^{*}\right)$. The locus $\rho_{\mathrm{W}}^{*}\left(T^{*}\right)$ is also plotted in Fig. 8. Although it extends to the region of oscillatory decay, the line $\rho_{\mathrm{W}}^{*}\left(T^{*}\right)$ is relevant only in the region of monotonic decay, i.e., below the $\mathrm{FW}$ line, where the asymptotic behavior is $h(r) \sim r^{-1} e^{-\kappa r}$ [see Eq. (17)]. In that region, the line $\rho_{\mathrm{W}}^{*}\left(T^{*}\right)$ marks the states where the correlation length $\xi=\kappa^{-1}$ presents a maximum at a given temperature, so that it can be termed a Widom line. In general, a Widom line refers to a locus of maximum response that ends at a critical point [20, 26, 45, 59, 70, 90, 91]. Thus, it represents an exten- 


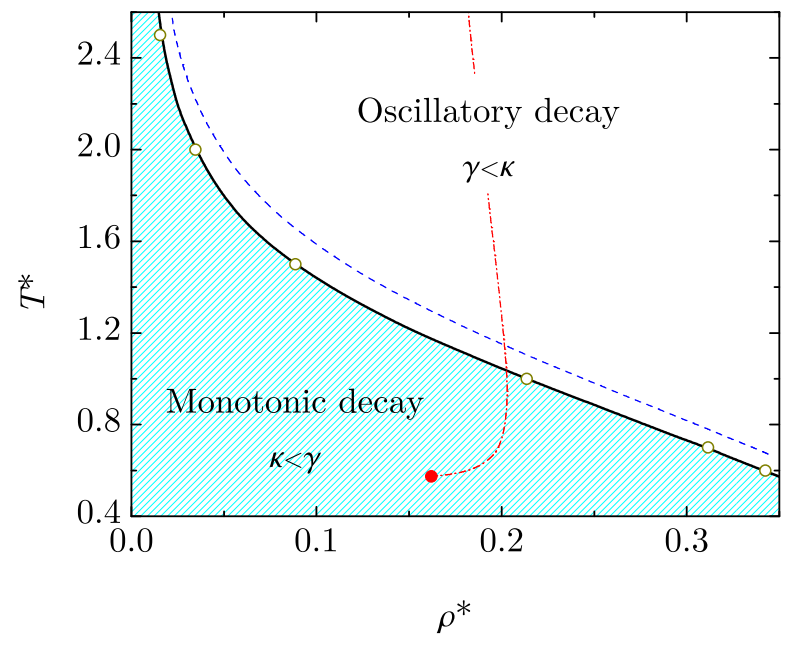

FIG. 8. Phase diagram in the $T^{*}-\rho^{*}$ plane for a fluid with a Jagla potential $\left(\lambda_{1}=1.3, \lambda_{2}=1.6, \varepsilon_{1} / \varepsilon_{2}=1\right)$, as predicted by the RFA $(n=10)$. The FW line (solid curve) splits the diagram into the hatched region where the decay of $h(r)$ is monotonic (i.e., $\kappa<\gamma$ ) and the region where the decay is oscillatory (i.e., $\gamma \leq \kappa$ ). The dash-dotted curve represents the Widom line, where, at a given temperature, $\kappa$ presents a minimum value. The Widom line inside the monotonic decay region terminates at the critical point (solid circle), where $\kappa \rightarrow 0$. The dashed curve is the FW line obtained from the RFA with $n=5$, while the open circles are points of the FW line obtained from the RFA with $n=20$.

sion of the coexistence line into the one-phase region. As shown in Fig. 7, the Widom point at $T^{*}=0.58$ corresponds to a value of $\kappa$ rather close to zero, so $T^{*}=0.58$ is only slightly above the critical temperature $T_{c}^{*}$ (where $\kappa \rightarrow 0)$. A more precise estimate of the critical point yields $T_{c}^{*}=0.574$ and $\rho_{c}^{*}=0.162$.

In order to assess the reliability of the RFA prediction for the FW line, we performed detailed MC simulations of the Jagla fluid (again with $\lambda_{1}=1.3, \lambda_{2}=1.6$, and $\varepsilon_{1} / \varepsilon_{2}=1$ ). While a pole analysis similar to the one performed above can also be carried out using simulation data as input [83], here we focus on the direct measurement of $h(r)$ for large $r$. In this instance, we took $N=5324$ particles and 2000 independent simulations for each physical state, divided into four blocks of 500 simulations each. In the first block, the system was aged for $10^{5} \mathrm{MC}$ steps to reach equilibration and then $2 \times 10^{5}$ additional MC steps were performed for data collection. The final equilibrated states of the first block were taken as the initial states for the second block, and so on. Along the equilibrated $2 \times 10^{5} \mathrm{MC}$ steps for each simulation, data were recorded every $50 \mathrm{MC}$ steps and averaged every 1000 saved configurations. The averaged function $g(r)$ was evaluated with a bin size $\delta r=0.01$.

We chose two representative states: (A) $\left(T^{*}, \rho^{*}\right)=$ $(2 / 3,0.2)$ and $(\mathrm{B})\left(T^{*}, \rho^{*}\right)=(2 / 3,0.4)$. According to Figs. 7 and 8 , the RFA predicts that state $\mathrm{A}$ lies in the monotonic-decay region, while state $\mathrm{B}$ lies in the oscillatory-decay region. These predictions are confirmed

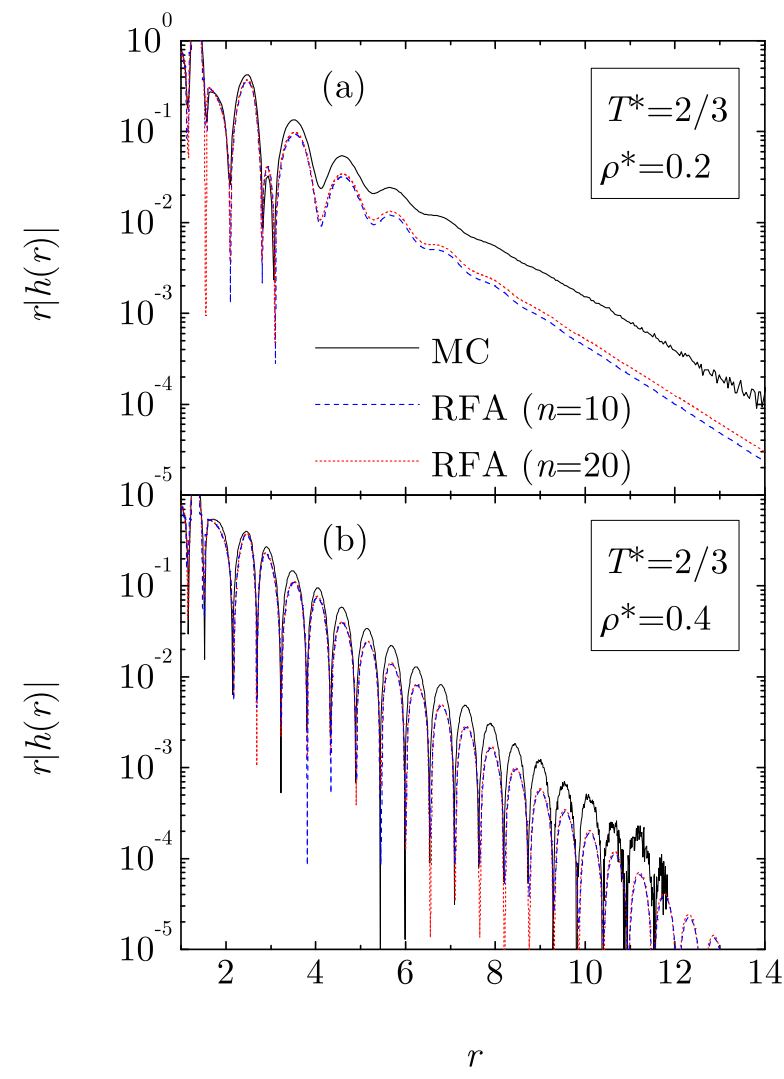

FIG. 9. Semilogarithmic plot of $r|h(r)|$ for a fluid with a Jagla potential $\left(\lambda_{1}=1.3, \lambda_{2}=1.6, \varepsilon_{1} / \varepsilon_{2}=1\right)$ at $\left(\right.$ a) $\left(T^{*}, \rho^{*}\right)=$ $(2 / 3,0.2)$ and (b) $\left(T^{*}, \rho^{*}\right)=(2 / 3,0.4)$. The solid, dashed, and dotted lines correspond to MC simulations, and to the RFA with $n=10$ and $n=20$, respectively.

by Fig. 9, which shows $r|h(r)|$ as measured in our MC simulations and as obtained from the RFA (with $n=10$ and $n=20$ ). It must be noted that, due to unavoidable finite-size effects, the asymptotic value $g_{\infty}$ of $g(r)$ in the MC simulations is slightly smaller than 1 , and this needs to be taken into account in the MC evaluation of $h(r)$ as $h(r)=g(r)-g_{\infty}$. In particular, $g_{\infty}=0.99912(1)$ and $g_{\infty}=0.99987(1)$ at states A and B, respectively. Therefore, the error in $h(r)$ is of order $10^{-5}$ and this is why the maximum accessible distance is $r \sim 10$, which corresponds to $r|h(r)| \sim 10^{-4}$.

Figure 9 not only confirms that the decay at states $\mathrm{A}$ and $\mathrm{B}$ is monotonic and oscillatory, respectively, but also that the RFA produces reasonable estimates of the damping coefficients $\gamma$ and $\kappa$, respectively. While the true values of $\gamma$ and $\kappa$ are somewhat smaller than the RFA values, Fig. 9(b) shows an excellent theoretical prediction of the wavelength $2 \pi / \omega$. Moreover, a very good agreement exists between the results of the RFA approach and the MC simulation data for the region prior to the asymptotic regime $(r \lesssim 4)$.

Next, we turn to another issue related to the asymptotic behavior of $h(r)$ for the Jagla fluid. The (static) structure factor $S(q)$ and the total correlation function 


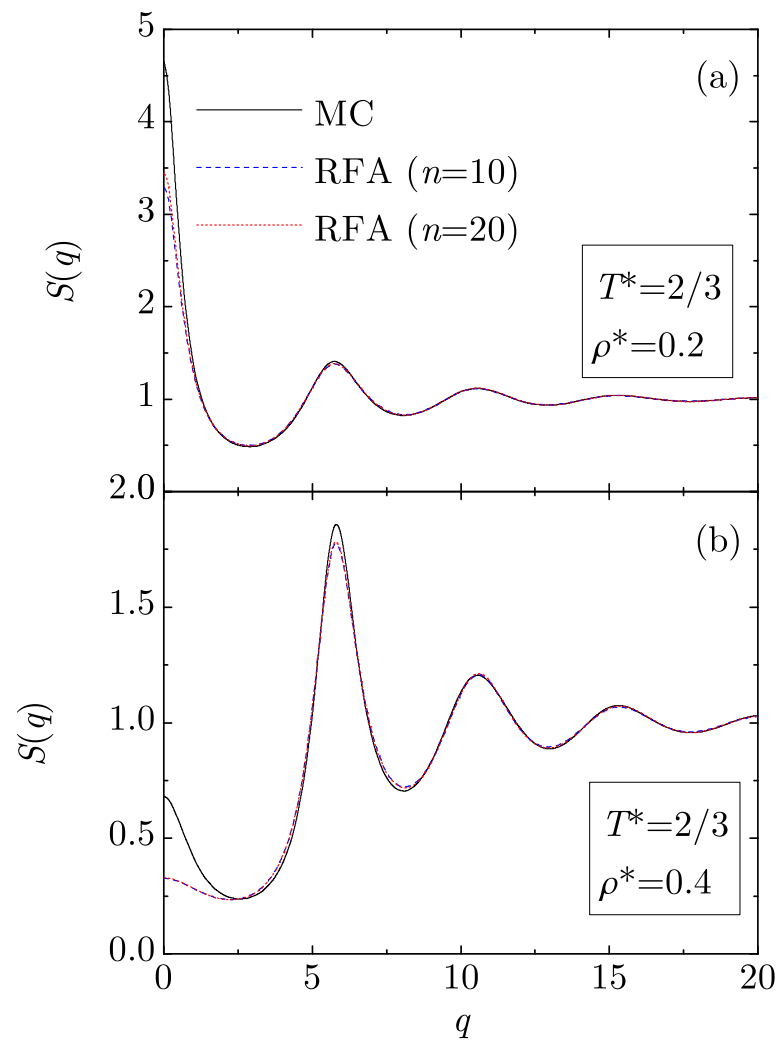

FIG. 10. Structure factor of a fluid with a Jagla potential $\left(\lambda_{1}=1.3, \lambda_{2}=1.6, \varepsilon_{1} / \varepsilon_{2}=1\right)$ at $(\mathrm{a})\left(T^{*}, \rho^{*}\right)=(2 / 3,0.2)$ and (b) $\left(T^{*}, \rho^{*}\right)=(2 / 3,0.4)$. The solid, dashed, and dotted lines correspond to MC simulations, and to the RFA with $n=10$ and $n=20$, respectively.

$h(r)$ are related by $[80,82$

$$
S(q)=1+\rho \widetilde{h}(q)
$$

where

$$
\begin{aligned}
\widetilde{h}(q) & =\int d \mathbf{r} e^{-\imath \mathbf{q} \cdot \mathbf{r}} h(r) \\
& =\frac{4 \pi}{q} \int_{0}^{\infty} d r r \sin (q r) h(r)
\end{aligned}
$$

is the Fourier transform of the total correlation function. Thus, it is easy to check the following exact relationship between $S(q)$ and $G(s)$ [80],

$$
S(q)=1-12 \eta\left[\frac{G(s)-G(-s)}{s}\right]_{s=\imath q} .
$$

Since $S(0)$ coincides with the (reduced) isothermal compressibility, the low- $q$ behavior of $S(q)$, which is deeply related to the asymptotic behavior of $h(r)$ at large $r$, provides us with another source for performing the evaluation of the RFA approach regarding thermodynamic properties. The structure factors for the same two cases A and B examined in Fig. 9 are shown in Fig. 10. The RFA curves were obtained from the analytic function $G^{(n)}(s)$ by application of Eq. (20), so they actually represent the discretized potential $\phi^{(n)}(r)$. However, we have checked that the "refined" structure factor obtained by making use of Eq. (5) is practically indistinguishable from the one obtained from $G^{(n)}(s)$. As for the $\mathrm{MC}$ curves, they were computed by a numerical integration using the MC values of $h(r)$ from $r=0$ up to a cutoff distance $r=r_{c} \approx 12$, plus the analytic integration from $r_{c}$ to infinity using the expressions of $h(r)$ at large $r$ given by Eq. (17), with a fit of $A_{\kappa}$ and $\kappa$ in case $\mathrm{A}$, and of $\left|A_{\gamma}\right|, \gamma, \omega$, and $\psi$ in case B.

In agreement with what is observed in Fig. 9, the RFA structure factors with $n=10$ and $n=20$ are practically indistinguishable from each other. Only a small increase of $S(0)$ (of about 3\%) can be seen in Fig. 10(a) when going from $n=10$ to $n=20$, thus reflecting a corresponding increase of the correlation length from $\xi=\kappa^{-1}=1.364$ to $\xi=\kappa^{-1}=1.398$. Regarding the comparison with the MC curves, a very good general agreement exists, except in the region $0 \leq q \lesssim 2$. In that region, for the two cases $\mathrm{A}$ and $\mathrm{B}$ considered in Figs. 9 and 10, $S(q)$ is rather sensitive to the asymptotic behavior of $h(r)$. On the other hand, although not shown, it is important to point out here that the agreement between the results of the RFA approach and the MC simulation data for the structure factor $S(q)$ is very good, even near $q=0$, for states (such as those considered in Fig. (4) with a relatively rapid decay of $h(r)$.

\section{CONCLUDING REMARKS}

The results of the previous sections deserve further consideration. By replacing the actual Jagla potential by a discretized version consisting of a hard-core and a suitable piecewise constant function, and assuming a common cavity function, we have been able to compute a semi-analytical RFA for the radial distribution function of the Jagla fluid, and also for the two limit cases of the triangle-well fluid and the ramp fluid. In the illustrative examples that we have presented, this leads to a highly satisfactory agreement between the results of the RFA approach and the MC data. Such agreement is in some instances superior to the one exhibited by the results of the PY and HNC integral equation approximations. Although not shown, we have found that the above performance of the RFA improves when the potential range $\lambda_{2}$ decreases. On the other hand, it becomes poorer as either the temperature decreases or the density increases. Since the number $n$ of steps involved in the discretization leads to feasible numerical solutions of Eq. (12), the method represents an excellent compromise between accuracy and simplicity. This is reinforced by the fact that going from $n=10$ to $n=20$ steps does not produce a significant difference in the numerical values of the radial distribution function.

The analysis of the asymptotic behavior of the total correlation function for large distances, as represented by 
the Fisher-Widom and Widom lines, also confirms that the RFA approach for the Jagla fluid is both simple and useful. In fact, it produces very reasonable estimates of the damping coefficients for either the monotonic or oscillatory behavior and, in this latter instance, it even leads to an excellent theoretical prediction of the wavelength. All of the above provides support to the idea that a similar approach to the one followed here for the Jagla fluid may be profitably employed to compute the structural properties of fluids whose molecules interact with other continuous potentials.

\section{ACKNOWLEDGMENTS}

We are grateful to R. Evans for proposing us the study of the Fisher-Widom line. Financial support of the Spanish Agencia Estatal de Investigación through Grant No. FIS2016-76359-P and the Junta de Extremadura (Spain) through Grant No. GR18079, both partially financed by FEDER funds, is acknowledged. M.L.H. thanks Universidad de Extremadura, where most of this work was carried out, and CONACYT (Mexico) for a sabbatical grant that made possible his stay there.
[1] P. C. Hemmer and G. Stell, "Fluids with several phase transitions," Phys. Rev. Lett. 24, 1284-1287 (1970).

[2] G. Stell and P. C. Hemmer, "Phase transitions due to softness of the potential core," J. Chem. Phys. 56, 4274-4286 (1972).

[3] P. G. Debenedetti, V. S. Raghavan, and S. S. Borick, "Splnodal curve of some supercooled liquids," J. Phys. Chem. 95, 4540-4551 (1991).

[4] G. Malescio, F. Saija, and S. Prestipino, "Anomalous melting behavior under extreme conditions: Hard matter turning "sof"," J. Chem. Phys. 129, 241101 (2009)

[5] S. Prestipino, F. Saija, and G. Malescio, "Anomalous phase behavior in a model fluid with only one type of local structure," J. Chem. Phys. 133, 144504 (2010).

[6] E. Lascaris, G. Malescio, S. V. Buldyrev, and H. E. Stanley, "Cluster formation, waterlike anomalies, and reentrant melting for a family of bounded repulsive interaction potentials," Phys. Rev. E 81, 031201 (2010).

[7] G. Malescio, S. Prestipino, and F. Saija, "Anomalous melting and solid polymorphism of a modified inversepower potential," Mol. Phys. 109, 2837-2844 (2011).

[8] G. Malescio and F. Saija, "A criterion for anomalous melting in systems with isotropic interactions," J. Phys. Chem. B 115, 14091-14098 (2011)

[9] E. A. Jagla, "Phase behavior of a system of particles with core collapse," Phys. Rev. E 58, 1478-1486 (1998).

[10] M. R. Sadr-Lahijany, A. Scala, S. V. Buldyrev, and H. E. Stanley, "Liquid-state anomalies and the Stell-Hemmer core-softened potential," Phys. Rev. Lett. 81, 4895-4898 (1998)

[11] E. A. Jagla, "Core-softened potentials and the anomalous properties of water," J. Chem. Phys. 111, 8980-8986 (1999)

[12] A. Scala, M. R. Sadr-Lahijany, N. Giovambattista, S. V. Buldyrev, and H. E. Stanley, "Applications of the Stell-Hemmer potential to understanding second critical points in real systems," J. Stat. Phys. 100, 97-106 (2000)

[13] A. Scala, M. R. Sadr-Lahijany, N. Giovambattista, S. V. Buldyrev, and H. E. Stanley, "Waterlike anomalies for core-softened models of fluids: Two-dimensional systems," Phys. Rev. E 63, 041202 (2001)

[14] N. B. Wilding and J. E. Magee, "Phase behavior and thermodynamic anomalies of core-softened fluids," Phys. Rev. E 66, 031509 (2002)

[15] E. A. Jagla, "The interpretation of water anomalies in terms of core-softened models,"
Braz. J. Phys. 34, 17-23 (2004).

[16] Z. Yan, S. V. Buldyrev, N. Giovambattista, and H. E. Stanley, "Structural order for one-scale and two-scale potentials," Phys. Rev. Lett. 95, 130604 (2005)

[17] P. Kumar, S. V. Buldyrev, F. Sciortino, E. Zaccarelli, and H. E. Stanley, "Static and dynamic anomalies in a repulsive spherical ramp liquid: Theory and simulation," Phys. Rev. E 72, 021501 (2005).

[18] A. B. de Oliveira, P. A. Netz, T. Colla, and M. C. Barbosa, "Structural anomalies for a three dimensional isotropic core-softened potential," J. Chem. Phys. 125, 124503 (2006)

[19] Z. Yan, S. V. Buldyrev, N. Giovambattista, P. G. Debenedetti, and H. E. Stanley, "Family of tunable spherically symmetric potentials that span the range from hard spheres to waterlike behavior," Phys. Rev. E 73, 051204 (2006).

[20] L. Xu, S. V. Buldyrev, C. A. Angell, and H. E. Stanley, "Thermodynamics and dynamics of the two-scale spherically symmetric Jagla ramp model of anomalous liquids," Phys. Rev. E 74, 031108 (2006).

[21] J. B. Caballero and A. M. Puertas, "Density anomaly and liquid-liquid transition from perturbation theories," Phys. Rev. E 74, 051506 (2006).

[22] G. Franzese, "Differences between discontinuous and continuous soft-core attractive potentials: The appearance of density anomaly," J. Mol. Liq. 136, 267-273 (2007).

[23] A. B. de Oliveira, G. Franzese, P. A. Netz, and M. C. Barbosa, "Waterlike hierarchy of anomalies in a continuous spherical shouldered potential," J. Chem. Phys. 128, 064901 (2008)

[24] A. B. de Oliveira, P. A. Netz, and M. C. Barbosa, "Which mechanism underlies the waterlike anomalies in core-softened potentials?" Eur. Phys. J. B 64, 481-486 (2008)

[25] A. B. de Oliveira, P. A. Netz, and M. C. Barbosa, "An ubiquitous mechanism for water-like anomalies," Europhys. Lett. 85, 36001 (2009).

[26] S. B. Buldyrev, G. Malescio, C. A. Angell, N. Giovanbattista, S. Prestipino, F. Saija, H. E. Stanley, and L. Xu, "Unusual phase behavior of onecomponent systems with two-scale isotropic interactions," J. Phys.: Condens. Matter 21, 504106 (2009)

[27] N. V. Gribova, Yu. D. Fomin, D. Frenkel, and V. N. Ryzhov, "Waterlike thermodynamic anomalies in a repulsive-shoulder potential system," Phys. Rev. E 79, 051202 (2009). 
[28] O. Pizio, H. Dominguez, Y. Duda, and S. Sokołowski, "Microscopic structure and thermodynamics of a coresoftened model fluid: Insights from grand canonical Monte Carlo simulations and integral equations theory," J. Chem. Phys. 130, 174504 (2009).

[29] F. Saija, S. Prestipino, and G. Malescio, "Anomalous phase behavior of a soft-repulsive potential with a strictly monotonic force," Phys. Rev. E 80, 031502 (2009).

[30] G. Franzese and H. E. Stanley, "Understanding the unusual properties of water," in Water and Life. The Unique Properties of $\mathrm{H}_{2} \mathrm{O}$, edited by R. M. Lynden-Bell, J. D. Barrow, S. C. Morris, J. L. Finney, and C. Harper (CRC Press, Boca Raton, 2010) pp. 101-118.

[31] O. Pizio, Z. Sokołowska, and S. Sokołowski, "Microscopic structure and thermodynamics of a core-softened model fluid from the second-order integral equations theory," Condens. Matter Phys. 14, 13601 (2011).

[32] P. Vilaseca and G. Franzese, "Isotropic soft-core potentials with two characteristic length scales and anomalous behaviour," J. Non-Cryst. Solids 357, 419-426 (2011).

[33] N. M. Barraz Jr., E. Salcedo, and M. C. Barbosa, "Thermodynamic, dynamic, structural, and excess entropy anomalies for core-softened potentials," J. Chem. Phys. 135, 104507 (2011).

[34] D. Nayar and C. Chakravarty, "Water and water-like liquids: relationships between structure, entropy and mobility," Phys. Chem. Chem. Phys. 15, 14162 (2013)

[35] M. P. Ciamarra and P. Sollich, "High-order jamming crossovers and density anomalies," Soft Matter 9, 9557-9561 (2013).

[36] L. Heckmann and B. Drossel, "Common features of simple water models," J. Chem. Phys. 138, 234503 (2013).

[37] M. Huš and T. Urbic, "Core-softened fluids as a model for water and the hydrophobic effect," J. Chem. Phys. 139, 114504 (2013)

[38] M. Huš and T. Urbic, "Thermodynamics and the hydrophobic effect in a core-softened model and comparison with experiments," Phys. Rev. E 90, 022115 (2014).

[39] M. Yasutomi, "Thermodynamic mechanism of the density anomaly of liquid water," Front. Phys. 3, 8 (2015)

[40] G. Munaò and F. Saija, "Density and structural anomalies in soft-repulsive dimeric fluids," Phys. Chem. Chem. Phys. 18, 9484-9489 (2016).

[41] G. Sun, L. Xu, and N. Giovambattista, "Relationship between the potential energy landscape and the dynamic crossover in a water-like monatomic liquid with a liquid-liquid phase transition," J. Chem. Phys. 146, 014503 (2017)

[42] M. Lukši, B. Hribar-Lee, and O. Pizio, "Phase behaviour of a continuous shouldered well model fluid. A grand canonical Monte Carlo study," J. Mol. Liq. 228, 4-10 (2017)

[43] S. Higuchi, D. Kato, D. Awaji, and K. Kim, "Connecting thermodynamic and dynamical anomalies of water-like liquid-liquid phase transition in the Fermi-Jagla model," J. Chem. Phys. 148, 094507 (2018).

[44] P. A. Netz, J. F. Raymundi, A. S. Camera, and M. C. Barbosa, "Dynamic anomalies of fluids with isotropic doubled-ranged potential," Physica A 342, 48-53 (2004).

[45] L. Xu, I. Ehrenberg, S. V. Buldyrev, and H. E. Stanley, "Relationship between the liquid-liquid phase transition and dynamic behaviour in the Jagla model," J. Phys.: Condens. Matter 18, S2239-S2246 (2006).
[46] L. Xu, F. Mallamace, Z. Yan, F. W. Starr, S. V. Buldyrev, and H. E. Stanley, "Appearance of a fractional Stokes-Einstein relation in water and a structural interpretation of its onset," Nat. Phys. 5, 565-569 (2009).

[47] Yu. D. Fomin, E. N. Tsiok, and V. N. Ryzhov, "Core-softened system with attraction: Trajectory dependence of anomalous behavior," J. Chem. Phys. 135, 124512 (2011).

[48] Yu. D. Fomin, E. N. Tsiok, and V. N. Ryzhov, "Silicalike sequence of anomalies in core-softened systems," Phys. Rev. E 87, 042122 (2013).

[49] S. V. Buldyrev and G. Franzese, "Two types of dynamic crossovers in a network-forming liquid with tetrahedral symmetry," J. Non-Cryst. Solids 407, 39-398 (2015).

[50] H. E. Stanley, C. A. Angell, U. Essmann, M. Hemmati, P. H. Poole, and F. Sciortino, "Is there a second critical point in liquid water?" Physica A 205, 122-139 (1994)

[51] E. Velasco, L. Mederos, G. Navascués, P. C. Hemmer, and G. Stell, "Complex phase behavior induced by repulsive interactions," Phys. Rev. Lett. 85, 122-125 (2000).

[52] E. A. Jagla, "Liquid-liquid equilibrium for monodisperse spherical particles," Phys. Rev. E 63, 061501 (2001)

[53] E. A. Jagla, "Low-temperature behavior of coresoftened models: Water and silica behavior," Phys. Rev. E 63, 061509 (2001).

[54] G. Franzese, G. Malescio, A. Skibinsky, S. V. Buldyrev, and H. E. Stanley, "Generic mechanism for generating a liquid-liquid phase transition," Nature 409, 692-695 (2001)

[55] G. Malescio and G. Pellicane, "Simple fluids with complex phase behavior," Phys. Rev. E 63, 020501(R) (2001).

[56] S. V. Buldyrev, G. Franzese, N. Giovambattista, G. Malescio, M. R. Sadr-Lahijany, A. Scala, A. Skibinsky, and H. E. Stanley, "Double-step potential models of fluids," in New Kinds of Phase Transitions: Transformations in Disordered Substances, NATO Science Series II. Mathematics, Physics and Chemistry, Vol. 81, edited by V. Brazhkin, S. V. Buldyrev, V. N. Ryzhov, and H. E. Stanley (Springer, 2002).

[57] V. N. Ryzhov and S. M. Stishov, "Repulsive step potential: A model for a liquid-liquid phase transition," Phys. Rev. E 67, 010201(R) (2003).

[58] A. Skibinsky, S. V. Buldyrev, G. Franzese, G. Malescio, and H. E. Stanley, "Liquid-liquid phase transitions for soft-core attractive potentials," Phys. Rev. E 69, 061206 (2004).

[59] L. Xu, P. Kumar, S. V. Buldyrev, S.-H. Chen, P. H. Poole, F. Sciortino, and H. E. Stanley, "Relation between the Widom line and the dynamic crossover in systems with a liquid-liquid phase transition," Proc. Natl. Acad. Sci. USA 102, 16558-16562 (2005).

[60] H. M. Gibson and N. B. Wilding, "Metastable liquidliquid coexistence and density anomalies in a coresoftened fluid," Phys. Rev. E 73, 061507 (2006).

[61] S. Zhou, A. Jamnik, E. Wolfe, and S. V. Buldyrev, "Local structure and thermodynamics of a coresoftened potential fluid: Theory and simulation," ChemPhysChem 8, 138-147 (2007).

[62] H. E. Stanley, P. Kumar, L. Xua, Z. Yan, M. G. Mazza, S. V. Buldyrev, S.-H. Chen, and F. Mallamace, "The puzzling unsolved mysteries of liquid water: Some recent progress," Physica A 386, 729-743 (2007)

[63] A. L. Benavides, L. A. Cervantes, and J. Torres, "Dis- 
crete perturbation theory for the Jagla ramp potential," J. Phys. Chem. C 111, 16006-16012 (2007)

[64] E. Lomba, N. G. Almarza, C. Martín, and C. McBride, "Phase behavior of attractive and repulsive ramp fluids: Integral equation and computer simulation studies," J. Chem. Phys. 126, 244510 (2007)

[65] Z Yan, S. V. Buldyrev, P. Kumar, N. Giovambattista, and H. E. Stanley, "Correspondence between phase diagrams of the TIP5P water model and a spherically symmetric repulsive ramp potential with two characteristic length scales," Phys. Rev. E 77, 042201 (2008)

[66] J. S. Høye, E. Lomba, and N. G. Almarza, "Oneand three-dimensional lattice models with two repulsive ranges: simple systems with complex phase behaviour," Mol. Phys. 107, 321-330 (2009).

[67] L. Xu, S. V. Buldyrev, N. Giovambattista, and H. E. Stanley, "Liquid-liquid phase transition and glass transition in a monoatomic model system," Int. J. Mol. Sci. 11, 5184-5200 (2010)

[68] M. Huš, G. Munaò, and T. Urbic, "Properties of a soft-core model of methanol: An integral equation theory and computer simulation study," J. Chem. Phys. 141, 164505 (2014).

[69] J. Luo, L. Xu, E. Lascaris, H. E. Stanley, and S. V. Buldyrev, "Behavior of the Widom line in critical phenomena," Phys. Rev. Lett. 112, 135701 (2014)

[70] J. Luo, L. Xu, C. A. Angell, H. E. Stanley, and S. V. Buldyrev, "Physics of the Jagla model as the liquid-liquid coexistence line slope varies," J. Chem. Phys. 142, 224501 (2015).

[71] G. Munaò and T. Urbic, "Structure and thermodynamics of core-softened models for alcohols," J. Chem. Phys. 142, 214508 (2015)

[72] L. A. Cervantes, G. Jaime-Muñoz, A. L. Benavides, J. Torres-Arenas, and F. Sastre, "Discrete perturbation theory for continuous soft-core potential fluids," J. Chem. Phys. 142, 114501 (2015)

[73] F. Ricci and P. G. Debenedetti, "A free energy study of the liquid-liquid phase transition of the Jagla two-scale potential," J. Chem. Sci. 129, 801-823 (2017)

[74] M. A. Anisimov, M. Duška, F. Caupin, L. E. Amrhein, A. Rosenbaum, and R. J. Sadus, "Thermodynamics of fluid polyamorphism," Phys. Rev. X 8, 011004 (2018).

[75] J. Y. Abraham, S. V. Buldyrev, and N. Giovambattista, "Liquid and glass polymorphism in a monatomic system with isotropic, smooth pair interactions," J. Phys. Chem. B 115, 14229-14239 (2011)
[76] C. A. Angell, "Forty years of silica simulations. Which way now?" Int. J. Appl. Glass Sci. 6, 3-14 (2015).

[77] C. A. Angell, "On the uncertain distinction between fast landscape exploration and second amorphous phase (ideal glass) interpretations of the ultrastable glass phenomenon," J. Non-Cryst. Solids 407, 246-255 (2015)

[78] A. Santos, S. B. Yuste, and M. López de Haro, "Rational-function approximation for fluids interacting via piece-wise constant potentials," Condens. Matter Phys. 15, 23602 (2012)

[79] A. Santos, S. B. Yuste, M. López de Haro, M. Bárcenas, and P. Orea, "Structural properties of fluids interacting via piece-wise constant potentials with a hard core," J. Chem. Phys. 139, 074503 (2013)

[80] A. Santos, A Concise Course on the Theory of Classical Liquids. Basics and Selected Topics, Lecture Notes in Physics, Vol. 923 (Springer, New York, 2016).

[81] A. L. Benavides and A. Gil-Villegas, "The thermodynamics of molecules with discrete potentials," Mol. Phys. 97, 1225-1232 (1999).

[82] J.-P. Hansen and I. R. McDonald, Theory of Simple Liquids, 3rd ed. (Academic, London, 2006).

[83] M. Dijkstra and R. Evans, "A simulation study of the decay of the pair correlation function in simple fluids," J. Chem. Phys. 112, 1449-1456 (2000)

[84] M. E. Fisher and B. Widom, "Decay of correlations in linear systems," J. Chem. Phys. 50, 3756-3772 (1969).

[85] S. Pieprzyk, A. C. Brańka, and D. M. Heyes, "Representation of the direct correlation function of the hardsphere fluid," Phys. Rev. E 95, 062104 (2017)

[86] R. Evans, J. R. Henderson, D. C. Hoyle, A. O. Parry, and Z. A. Sabeur, "Asymptotic decay of liquid structure: oscillatory liquid-vapour density profiles and the FisherWidom line," Mol. Phys. 80, 755-775 (1993)

[87] C. Vega, L. F. Rull, and S. Lago, "Location of the FisherWidom line for systems interacting through short-ranged potentials," Phys. Rev. E 51, 3146-3155 (1995).

[88] W. E. Brown, "The Fisher-Widom line for a hard core attractive Yukawa fluid," Mol. Phys. 88, 579-584 (1996).

[89] P. Tarazona, E. Chacón, and E. Velasco, "The FisherWidom line for systems with low melting temperature," Mol. Phys. 101, 1595-1603 (2003).

[90] G. Ruppeiner, N. Dyjack, A. McAloon, and J. Stoops, "Solid-like features in dense vapors near the fluid critical point," J. Chem. Phys. 146, 224501 (2017)

[91] M. Raju, D. T. Banuti, P. C. Ma, and M. Ihme, "Widom lines in binary mixtures of supercritical fluids," Sci. Rep. 7, 3027 (2017). 